\title{
COVID-19 surveillance for all newborns at the NICU; conditio sine qua non?
}

\author{
J. Peter de Winter ${ }^{1,2}$ - Daniele De Luca ${ }^{3,4}$ - David G. Tingay ${ }^{5,6,7}$ \\ Published online: 12 August 2020 \\ (C) Springer-Verlag GmbH Germany, part of Springer Nature 2020
}

Previous studies have reported fewer cases of Coronavirus disease 2019 (COVID-19) in children than in adults, and to date there is limited literature about the clinical course and outcomes in neonates [1,2]. Not inappropriately the focus of COVID-19 therapies has been in adults, especially the aged. However, it is clear that an immature immune system makes neonates, especially those in the NICU, highly vulnerable to infections generally [3, 4], and thus probably also for COVID19. One case series has suggested that the younger the child, the higher risk of severe COVID-19 disease [5]. The first report of a neonate with COVID-19 infection was a 17 days old boy who recovered [6]. At the time of writing, complete data $^{1}$ on 35 neonates have been included in the database EPICENTRE [7]. Vertical risk was present in 33 neonates, and only two infants were reported to have community exposure. As more neonates are included in well-designed databases, the more we understand the risks, clinical spectrum, severity and complications in this patient population. Moreover, we can focus on ways to prevent COVID-19 infections in neonates born from an infected mother, or neonates that are infected in the community, whether at home, in hospital, by health care workers or families, especially during any potential new pandemic waves.

\footnotetext{
${ }^{1}$ Only neonates with a closed data entry have been included, this also does not include regions/countries submitting complete population datasets rather than via individual sites
}

See related article: https://doi.org/10.1007/s00431-020-03765-7

J. Peter de Winter ejp@spaarnegasthuis.nl

1 Department of Pediatrics, Spaarne Gasthuis, Hoofddorp/ Haarlem, The Netherlands

2 Department of Development and Regeneration, KU Leuven, Leuven, Belgium

3 Division of Pediatrics and Neonatal Critical Care, "A. Beclere" medical center, Paris Saclay University Hospitals, Paris, France
Since the start of the pandemic, there has been an exponential increase in publications describing the measures that need to be taken in specific patient groups and this has resulted in various, and often diverse, proposals for guidelines [8]. Unfortunately, at the time of writing, there is no universally applicable, evidence-based guideline for the prevention and management of COVID-19 infection in the paediatric population,. Evidence-based policies are needed on to prevention of horizontal COVID-19 clusters within the NICU, as well as many other open questions. What changes in vulnerability may occur in neonates with different pre-morbid conditions, such as prematurity or cardiac disease? Is co-horting cases better? Should we test breastmilk more often? How do we manage or avoid contact with non-parental relatives and siblings, or should they be tested before their visits? How to handle rooming in and post-discharge care?

For the time being, we have to assemble reasonable experience based guidelines to prevent COVID-19 within the NICU. In this issue of European Journal of Pediatrics, we publish an intriguing study performed in a $3^{\text {rd }}$ level Italian NICU located in one of the regions most affected by the pandemic [9]. The authors present a method to care for these vulnerable neonates. Cavicchiolo et al. describe their

4 Physiopathology and Therapeutic Innovation Unit, INSERM U999, Paris Saclay University, Paris, France

5 Neonatal Research, Murdoch Children's Research Institute, Melbourne, VIC, Australia

6 Department of Neonatology, Royal Children's Hospital, Melbourne, VIC, Australia

7 Department of Pediatrics, University of Melbourne, Melbourne, VIC, Australia 
experience with early heightened surveillance of all neonates during the peak two months of the initial pandemic period in their area. No positive tests in the group of 75 neonates admitted to their unit were found; however, five asymptomatic adult individuals were discovered.

Let's have a look at these vulnerable small neonates in the NICU. We know that pregnant women and their foetuses represent a high-risk group during COVID-19 outbreaks [1]. Initial reports suggest neonatal mortality was $2 \%[5,6]$ in mothers with COVID-19 in their third trimester, and $1 / 3$ of births were transferred to the NICU, mainly due to the need for investigations and monitoring. Now we have clear evidence that SARS-CoV-2 replicates in placental cells and that vertical intra-utero transmission can occur, although it remains a rare event. The same applies for transmission during delivery and an international classification to diagnose fetal and neonatal COVID is now available [10-12] Cavicchiolo's study was not focused on this group of potentially infected neonates, but the experience of neonates within a NICU. They followed all caregivers, caretakers and neonates during their stay at the NICU whilst standard hygiene precautions and appropriate use of protective equipment were being applied. Most neonates were already isolated in an incubator, a potentially under-appreciated barrier in the COVID-19 pandemic that is familiar to neonatal clinicians but not in other critical care settings. The five asymptomatic COVID-19 carriers had already had contact with inpatient neonates at the time of diagnosis. Several aspects have to be discussed in relation to this study. For obvious ethical reasons, this study was performed without a control ward. We never will know whether or not this surveillance would have prevented illnesses in the infants nor whether the increased COVID-19 hygiene measures had been enough without putting adults into quarantine. The psychological burden of increased hygiene measures and restricted access, in addition to the anxiety of being positive for COVID-19 might disrupt parental interaction and prevent health care worker well-being and performance.

Finally, the economic burden of this surveillance study should be investigated and compared with other measures and different NICU's. Time will tell whether the proposed, and frequently advised, measures of triage and adherence to appropriate protective equipment will decrease the burden of COVID-19 disease on the neonatal ward. or whether there exists a place for extreme measures (universal surveillance and banning family visits) have any role during sharp rises in community COVID-19 rates. Hopefully, several extreme measures will not be needed, especially if very rapid and reliable tests become available. For the time being, it seems reasonable to use the available published COVID-19 NICU protocols [13-15], and increase surveillance when case-rates rise locally; unfortunately probably a necessary condition we will continue to face for some time.

\section{Compliance with ethical standards}

Competing interests DDL and DGT are principal investigators of the European Society of Paediatric and Neonatal Intensive Care COVID19 Paediatric and Neonatal Registry (EPICENTRE Project). DGT is a deputy chair of the Australian National COVID-19 clinical evidence taskforce paediatric and adolescent panel. There are no other competing interests to declare.

Financial Information DGT is supported by a National Health and Medical Research Council Clinical Career Development Fellowship (Grant ID 1053889) and the Victorian Government Operational Infrastructure Support Program (Melbourne, Australia).

\section{References}

1. Marim F, Karadogan D, Eyuboglu TS et al (2020) Lessons Learned so Far from the Pandemic: A Review on Pregnants and Neonates with COVID-19. Eurasian J Med 52(2):202-210. https://doi.org/ 10.5152/eurasianjmed.2020.20118

2. Liguoro I, Pilotto C, Bonanni M et al (2020) SARS-COV-2 infection in children and newborns: a systematic review. Eur J Pediatr 179(7):1029-1046. https://doi.org/10.1007/s00431-020-03684-7

3. van Well GTJ, Daalderop LA, Wolfs T, Kramer BW (2017) Human perinatal immunity in physiological conditions and during infection. Mol Cell Pediatr 4(1):4. https://doi.org/10.1186/s40348-0170070-1

4. Ludvigsson JF (2020) Systematic review of COVID-19 in children shows milder cases and a better prognosis than adults. Acta Paediatr 109(6):1088-1095. https://doi.org/10.1111/apa.15270

5. Dong Y, Mo X, Hu Y et al (2020) Epidemiological Characteristics of 2143 Pediatric Patients With 2019 Coronavirus Disease in China. Pediatrics e20200702. https://doi.org/10.1542/peds.20200702

6. Ovali F (2020) SARS-CoV-2 Infection and the newborn. Front Pediatr 8:294. https://doi.org/10.3389/fped.2020.00294

7. De Luca D, Rava L, Nadel S et al (2020) The EPICENTRE (ESPNIC Covid pEdiatric Neonatal Registry) initiative: background and protocol for the international SARS-CoV-2 infections registry. Eur J Pediatr 179(8):1271-1278. https://doi.org/10.1007/ s00431-020-03690-9

8. Yeo KT, Oei JL, De Luca D, Schmölzer GM, Guaran R, Palasanthiran P, Kumar K, Buonocore G, Cheong J, Owen LS, Kusuda S, James J, Lim G, Sharma A, Uthaya S, Gale C, Whittaker E, Battersby C, Modi N, Norman M, Naver L, Giannoni E, Diambomba Y, Shah PS, Gagliardi L, Harrison M, Pillay S, Alburaey A, Yuan Y, Zhang H (2020) Review of guidelines and recommendations from 17 countries highlights the challenges that clinicians face caring for neonates born to mothers with COVID-19. Acta Paediatr. https://doi.org/10.1111/apa.15495

9. Cavicchiolo ME, Trevisanuto D, Lolli E, Mardegan V, Saieva AM, Franchin E, Plebani M, Donato D, Baraldi E (2020) Universal screening of high-risk neonates, parents and staff at a neonatal intensive care unit during the SARS-CoV-2 pandemic. Eur J Pediatr. https://doi.org/10.1007/s00431-020-03765-7

10. Vivanti AJ, Vauloup-Fellous C, Prevot S, Zupan V, Suffee C, Do Cao J, Benachi A, De Luca D (2020) Transplacental transmission of SARS-CoV-2 infection. Nat Commun 11(1):3572. https://doi.org/ 10.1038/s41467-020-17436-6

11. Raschetti R, Vivanti A, Vauloup C, Loi B, Benachi A, De Luca D (2020) Neonatal SARS-CoV-2 infections: systematic review, synthesis and meta-analysis of reported cases. 10.21203/rs.3.rs-48185/ 
v1. Preprint on https://www.researchsquare.com/article/rs-48185/ v1. Under review for Nature

12. Shah PS, Diambomba Y, Acharya G, Morris SK, Bitnun A (2020) Classification system and case definition for SARS-CoV-2 infection in pregnant women, fetuses, and neonates. Acta Obstet Gynecol Scand 99:565-568. https://doi.org/10.1111/aogs.13870

13. Shalish W, Lakshminrusimha S, Manzoni P, Keszler M, Sant'Anna GM (2020) COVID-19 and Neonatal Respiratory Care: Current Evidence and Practical Approach. Am J Perinatol 37(8):780-791. https://doi.org/10.1055/s-0040-1710522

14. Juan J, Gil MM, Rong Z, Zhang Y, Yang H, Poon LC (2020) Effect of coronavirus disease 2019 (COVID-19) on maternal, perinatal and neonatal outcome: systematic review. Ultrasound Obstet Gynecol 56(1):15-27. https://doi.org/10.1002/uog.22088

15. Verma S, Lumba R, Lighter JL et al (2020) Neonatal intensive care unit preparedness for the Novel Coronavirus Disease-2019 pandemic: A New York City hospital perspective. Curr Probl Pediatr Adolesc Health Care 50(4):100795. https://doi.org/10.1016/j. cppeds.2020.100795

Publisher's note Springer Nature remains neutral with regard to jurisdictional claims in published maps and institutional affiliations. 